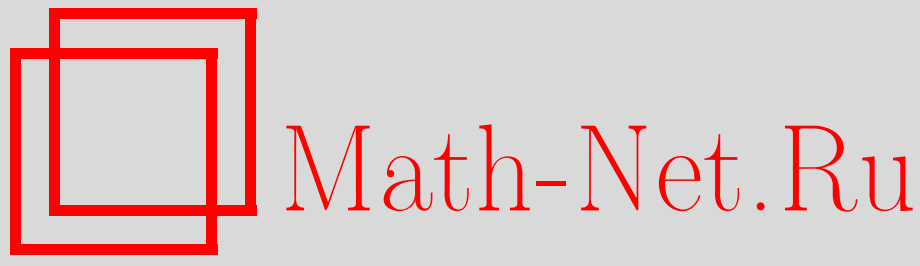

Общероссийский математический портал

Ю. Г. Леонов, О нижней оценке функции роста группы Григорчука, Матем. заметки, 2000, том 67, выпуск 3, 475-477

DOI: https://doi.org/10.4213/mzm860 
Использование Общероссийского математического портала Math-Net.Ru подразумевает, что вы прочитали и согласны с пользовательским соглашением http://www. mathnet.ru/rus/agreement

Параметры загрузки:

IP: 54.162 .27 .143

26 апреля 2023 г., 15:47:07

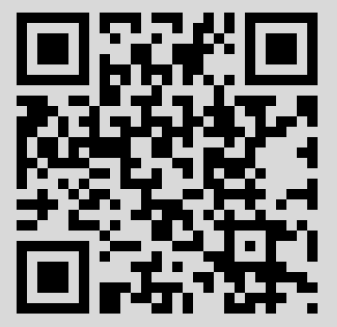




\section{О НИЖНЕЙ ОЦЕНКЕ ФУНКЦИИ РОСТА ГРУППЫ ГРИГОРЧУКА}

\section{Ю.Г. Леонов}

Одной из самых известных периодических не локально конечных групп является 2-группа, построенная в 1980 году Р. И. Григорчуком [1]. Эта группа обладает также многими другими интересньми свойствами (см. [2], [3]), одним из которых является ее промежуточный рост.

Напомним, что функция роста конечно-порожденной группы $G$ с системой порождающих $S$ определяется соотношением

$$
\gamma(n)=\#\{g \in G ; l(g) \leqslant n\},
$$

где $l(g)$ - длина элемента $g$ относительно $S$.

Будем говорить, что функция $f_{1}(n)$ растет не быстрее, чем $f_{2}(n): f_{1}(n) \preccurlyeq f_{2}(n)$, если найдется $c>0$ такое, что $f_{1}(n) \leqslant f_{2}(c n)$ для любых $n \in \mathbb{N}$. Если $f_{1}(n) \preccurlyeq f_{2}(n)$ и $f_{2}(n) \preccurlyeq f_{1}(n)$, то функции эквивалентны: $f_{1}(n) \sim f_{2}(n)$. Функции роста одной и той же конечно-порожденной группы при различных конечных системах порождающих эквивалентны.

В 1968 году Дж. Милнор [4] поставил вопрос о существовании групп, у которых функция роста растет быстрее любой степенной и медленнее показательной. Такие группы называются группами промежуточного роста.

В работе [5] Григорчук показал, что группа Gr из [1] имеет промежуточный рост, а именно справедливы неравенства: $e^{\sqrt{n}} \preccurlyeq \gamma_{\mathrm{Gr}}(n) \preccurlyeq e^{n^{\log _{32} 31}}$, тем самым ответив на вопрос Милнора.

Продолжая исследовать шкалу роста конечно-порожденных групп в работе [6] Григорчук установил, что если группа аппроксимируется конечными $p$-группами и допускает оценку роста $\gamma_{G}(n) \prec e^{\sqrt{n}}$ (т.е. $f_{G}(n) \preccurlyeq e^{\sqrt{n}}$, а обратное неверно), то $G$ почти нильпотентна.

(C) Ю.Г. ЛЕонов 
Таким образом, для этого класса групп мы имеем шкалу степеней роста групп с лакуной между степенными функциями и функцией $e^{\sqrt{n}}$, причем Григорчуком высказана гипотеза о том, что эта лакуна даже имеет место для всего класса конечно-порожденных групп.

В связи с этим возник вопрос о существовании группы с функцией роста, эквивалентной $e^{\sqrt{n}}$. В работе [6] было установлено, что такие группы, при условии аппроксимации их конечными $p$-группами, имеют конечную ширину (т.е. ранги факторов нижнего центрального ряда равномерно ограниченны). Группы конечной шерины - это интересный и важный класс групп [7], и из известных конечно-порожденных групп промежуточного роста группа Григорчука является пока единственньм примером группы конечной ширины (другие примеры подобного рода из [8] родственны этой группе).

В работе [9] был поставлен вопрос о том: действительно ли эти группы имеют рост $e^{\sqrt{n}}$ ? В данной работе мы отвечаем на вопрос Григорчука отрицательно. А именно

ТеоремА. Для группь Григориука $G$ верна оченка

$$
\gamma_{G}(n) \succcurlyeq e^{n^{\alpha}}
$$

əде $\alpha=\log _{87 / 22} 2=0.506 \ldots>1 / 2$.

Прежде чем сформулировать основные вспомогательные леммы, используемые при доказательстве теоремы, определим группу $G$, а также отметим основные ее свойства.

Изначально группа Григорчука из [1] была определена им как группа преобразований отрезка $[0,1]$, сохраняющих лебегову меру. Позже выяснилось, что она имеет много других, эквивалентных заданий, например, как группа, порожденная конечными автоматами, или как группа, действующая автоморфизмами бинарного дерева. Здесь нам удобно интерпретировать ее элементы как преобразования в пространстве $\Omega=\{0,1\}^{\mathbb{N}}$ бесконечных (вправо) последовательностей алфавита $\{0,1\}$.

А именно, для любой последовательности $\alpha=\left\{\alpha_{0}, \alpha_{1}, \ldots\right\}$ определим преобразование $a$ по правилу

$$
a(\alpha)=\left\{\bar{\alpha}_{0}, \alpha_{1}, \ldots\right\}, \quad \text { где } \bar{x}= \begin{cases}0, & x=1 \\ 1, & x=0 .\end{cases}
$$

Пусть $\sigma(\alpha)=\left\{\alpha_{1}, \alpha_{2}, \ldots\right\}$ - сдвиг влево последовательности $\alpha$. Определим также преобразования $b, c, d$ по правилу: для любых $\alpha \in \Omega$ полагаем

$$
b(\alpha)=\left\{\begin{array}{ll}
0 a(\sigma(\alpha)), & \alpha_{0}=0, \\
1 c(\sigma(\alpha)), & \alpha_{0}=1,
\end{array} \quad c(\alpha)=\left\{\begin{array}{ll}
0 a(\sigma(\alpha)), & \alpha_{0}=0, \\
1 d(\sigma(\alpha)), & \alpha_{0}=1,
\end{array} \quad d(\alpha)= \begin{cases}\alpha, & \alpha_{0}=0, \\
1 b(\sigma(\alpha)), & \alpha_{0}=1 .\end{cases}\right.\right.
$$

Группа $G$ порождается элементами $a, b, c, d$ c операцией суперпозиции преобразований. Очевидно, $a^{2}=b^{2}=c^{2}=d^{2}=b c d=1$.

Пусть $H \leqslant G$ - подгруппа группы $G$, состоящая из элементов, оставляющих на месте первую букву любой последовательности $\alpha \in \Omega$. Легко видеть, что в $H$ входят те и только те слова группы $G$, которые имеют четное число букв $a$ в своей записи, и что $H=\langle b, c, d, a b a, a c a, a d a\rangle$.

Рассмотрим вложение (мономорфизм) $\psi: H \rightarrow G \times G$, заданное на порождающих следующим образом:

$$
\begin{gathered}
\psi(b)=(a, c), \quad \psi(c)=(a, d), \quad \psi(d)=(1, b), \\
\psi(a b a)=(c, a), \quad \psi(a c a)=(d, a), \quad \psi(a d a)=(b, 1)
\end{gathered}
$$

(это вложение было определено в [1]). Обозначим через $l(g)$ длину элемента $g \in G$ относительн системы порождающих $S=\{b, c, d, a b a, a c a, a d a\}$. Основным утверждением при доказательстве нашей теоремы является следующая

ЛЕмма 1. Пусть $g \in H, \psi(g)=\left(g_{0}, g_{1}\right), l\left(g_{0}\right), l\left(g_{1}\right) \geqslant R(=22)$ и пусть сущ,ествует $c>0$ с условием

$$
\left|l\left(g_{0}\right)-l\left(g_{1}\right)\right| \leqslant c \cdot \min \left\{l\left(g_{0}\right), l\left(g_{1}\right)\right\} .
$$


Тогда найдется число $Q<2$ такое, что

$$
l(g) \leqslant Q\left(l\left(g_{0}\right)+l\left(g_{1}\right)\right)+C_{1},
$$

$C_{1}$ - постоянная.

Отметим, что похожее утверждение при $Q=2$ является почти очевидньм (см. [5]).

Распространим $\psi$ на всю группу $G$, положив при $g \notin H \psi(g)=\psi(g a)$. Можно считать $\psi^{n}(g)$ упорядоченной последовательностью из $2^{n}$ элементов, определенной индукцией по правилу $\psi^{n}(g)=\left(\psi^{n-1}\left(g_{0}\right), \psi^{n-1}\left(g_{1}\right)\right)$. Из (1) легко следует, что если $\psi(g)=\left(g_{0}, g_{1}\right)$, то $l\left(g_{i}\right)<l(g)$, $i=0,1$, кроме случая, когда $g$ или $g a$ порождающий или единичный элемент. Таким образом, для любых $g \in G$ найдется $m$ такое, что любое слово в последовательности $\psi^{m}(g)$ имеет длину $\leqslant 1$. Наименьшее $m$ с таким свойством назовем глубиной и обозначим $f(g)$. Важную роль в оценке роста играет функция $r: \mathbb{N} \cup\{0\} \rightarrow \mathbb{N} \cup\{0\}$, определенная следующим образом:

$$
r(n)=\max _{g, f(g) \leqslant n} l(g) .
$$

Из леммы 1 выводится

Лемма 2. Существует $M<4$ такое, что для некоторой постоянной $c>0$ выполняemcя $r(n) \leqslant M \cdot r(n-1)+C$ для любых $n \in \mathbb{N}$.

Покажем, как, используя лемму 2 , получить утверждение теоремы. Рассмотрим множество $F(n)=\{g \in G ; f(g) \leqslant n\}$ и его мощность $|F(n)|=\#\{g \in G ; f(g) \leqslant n\}$. Можно показать (см. [10] или [11]), что

$$
|\bar{F}(n)| \geqslant 2^{2^{n} \cdot c_{1}}, \quad 0<c_{1} \leqslant 1 .
$$

Из леммы 2 следует, что $r(n) \leqslant M^{n} \cdot c^{\prime}$ и так как количество элементов длины $\leqslant r(n)$ не меньше, чем количество элементов в множестве $F(n)$, то

$$
\gamma_{G}(r(n)) \geqslant \bar{F}(n) \geqslant 2^{2^{n} \cdot c_{1}}
$$

и отсюда $\gamma_{G}(n) \succcurlyeq e^{n^{\alpha}}$, где $\alpha=\log _{M} 2$ и эта оценка доказывает теорему.

Отметим, что Л. Бартольди, используя основную идею автора, предложил недавно иное доказательство нашей теоремы [12].

Автор выражает глубокую благодарность Р. И. Григорчуку за постановку задачи и ценные замечания.

\section{СПИСОК ЦИТИРОВАННОЙ ЛИТЕРАТУРЫ}

1. Григорчук Р. И. // Функцион. анализ и его прилож. 1980. Т. 14. №1. С. 53-54. 2. Григорчук Р. И. // Матем. сб. 1998. Т. 189. №1. С. 79-100. 3. de la Harpe P. Topics in geometric group theory // Preprint at http://www . unige.ch/math/biblio/preprint/pp98.html, 1998. 4. Milnor J. // Amer. Math. Monthly. 1968. V. 75. №6. Р. 685-686. 5. Григорчук Р. И. // Изв. АН СССР. Сер. Матем. 1984. № 5. С. 939-985. 6. Григорчук Р. И. // Матем. сб. 1989. Т. 180. №2. C. 207-225. 7. Klaas G., Leedham-Green C. R., Plesken W. Linear Pro-p-groups of Finite Width. Lecture Notes in Math. V. 1674, 1996. 8. Bartholdi L., Grigorchuk R. I. Lie methods in growth of groups and groups of finite width. Preprint, 1999. 9. Grigorchuk R. I. // Proc. Int. Congress of Math. (Kyoto, 1990). V. I. Tokyo: Math. Soc. Japan, 1991. P. 325-338. 10. Рожков А. В. Условия конечности в группах автоморфизмов деревьев. Дисс. ... д. ф.-м.н. Челябинск: Челябинский гос. ун-т, 1996. 11. Леонов Ю. Г. // Матем. заметки. 1998. Т. 64. № 4. C. 573-583. 12. Bartholdi L. The growth of Grigorchuk's torsion group. Preprint. 